\title{
Response to chemotherapy and association with three tumour markers in breast cancer patients in Ghana
}

\author{
Emmanuel Amankwaa-Frempong1, Francis Agyemang Yeboah¹, Samuel Blay Nguah², \\ Osei Owusu Afriyie ${ }^{3}$ \\ ${ }^{I}$ Department of Molecular Medicine, University of Science and Technology, Kumasi, Ghana. \\ ${ }^{2}$ Department of Child Health, Komfo Anokye Teaching Hospital, Kumasi, Ghana. \\ ${ }^{3}$ Department of Pathology, Komfo Anokye Teaching Hospital, Kumasi, Ghana.
}

Received March 10, 2014; Revised May 21, 2014; Accepted May 22, 2014; Published Online June 07, 2014

\section{Original Article}

\begin{abstract}
Purpose: Oestrogen receptor (ER), progesterone receptor (PR) and human epidermal growth factor 2 (HER2/neu) expression in breast cancer patients predict response to chemotherapy though recorded extent vary. This retrospective study aimed to investigate the relationship between ER, PR and HER2/neu expression and response of breast cancer to chemotherapy at a tertiary hospital in Ghana. Methods: Records of all breast cancer cases seen from 2009 through 2011 were reviewed. Their receptor status, first line treatment [4 cycles of Adriamycin $\left(60 \mathrm{mg} / \mathrm{m}^{2}\right)+$ Cyclophosphamide $\left(600 \mathrm{mg} / \mathrm{m}^{2}\right)$ ], second line treatment [Capecitabine $\left(1 \mathrm{~g} / \mathrm{m}^{2}\right)+$ Paclitaxel $\left.\left(170 \mathrm{mg} / \mathrm{m}^{2}\right)\right]$ and clinical response were extracted. Results: Complete remission after first and second line treatments were observed in 36 (38.3\%, 95\% CI: 28.5 to 48.9 ) and 34 (58.6\%, 95\% CI: .44.9 to 71.4) respectively. After both first and second line treatment 70 (74.5\%, 95\% CI: 64.4 - 82.9) had gone into remission. Prevalence of ER, PR, HER2/neu and Triple negative breast cancer (TNBC) were $34.0 \%$ (95\% CI: 24.6 to 44.5 ), $20.2 \%$ (95\% CI: 12.6 to 29.7 ), $8.5 \%$ (95\% CI: 3.7 to $16.1)$ and 59.6\% (95\%CI: 48.9 to 69.6) respectively. ER and PR positivity were independently associated with complete remission after first line treatment while TNBC was associated with non-remission. Conversely ER was independently associated with non-remission after second line treatment while TNBC was associated with complete remission. Conclusion: ER and TNBC status are significant predictors of complete remission and non-remission respectively after chemotherapy for breast cancer patient in Ghana.
\end{abstract}

Keywords: Tumor Markers; Chemotherapy Response; Estrogen Receptors; HER/2neu; Progesterone Receptors

\section{Introduction}

Breast cancer accounted for $23 \%$ of the total new cancer cases in the world in 2008, making it the leading cause of cancer deaths in females. ${ }^{1}$ According to the Global cancer statistics in 2002, breast cancer was the most prevalent cancer in the world. ${ }^{2}$ Increased use of mammography screening and early detection seems to have contributed to the decline in the mortality rates in the developed world. ${ }^{3}$ However the

Corresponding author: Emmanuel Amankwaa-Frempong; Department of Molecular Medicine, School of Medical Sciences-Kwame Nkrumah, University of Science and Technology, Kumasi, Ghana.

Cite this article as:

Amankwaa-Frempong E, Yeboah FA, Nguah SB, Afriyie OO. Response to chemotherapy and association with three tumour markers in breast cancer patients in Ghana. Int J Cancer Ther Oncol 2014; 2(3):02034. DOI: 10.14319/ijcto.0203.4 story in the developing world may be different where the incidence and mortality rates are rising with younger women been diagnosed of the disease. ${ }^{4}$

In Ghana the age group commonly affected is between 40-49 years. ${ }^{5}$ Due to lack of education and effective screening measures patients often present to the hospital late with advanced stages of the disease. ${ }^{5}$ There are reports of unsatisfactory emotional support, communication and counselling for the breast cancer patients in Ghana, thereby making them resort to spiritualist and herbalist for support and treatment. ${ }^{6,7}$ There is an association between age and the histological type of the breast cancer in Ghanaian women, and the risk of the disease also increases after the age of 30 years. ${ }^{7}$ Many studies have been done on HER2/neu (an epidermal growth factor receptor) as a predictive marker for most chemotherapy regimens. There are conflicting reports on the 
relationship between HER2/neu and response to neo-adjuvant anthracycline based chemotherapy ${ }^{8}$ making it difficult to use to determine chemotherapy treatment options. However according to Muss et al., the benefit of an anthracycline based chemotherapy for breast cancer may be dependent on the over expression of HER2/neu receptors. ${ }^{9}$ Available data reveal that HER2/neu over-expression is associated with poor overall prognosis, shorter relapse-free and overall survival time, and resistance to adjuvant anthracycline chemotherapy. ${ }^{10-12}$ HER2/neu over-expression is associated with the dose-intensity and dose-density of an anthracycline based chemotherapy. Thor et al., showed that regimens of increased dose per cycle (dose intensity) of an anthracycline based chemotherapy are associated with an improved outcome only in women with HER2/neu over expression. ${ }^{13}$ Also, HER2/neu is now the standard predictive marker for transtuzumab (a humanized monoclonal antibody) in the treatment of breast cancer. ${ }^{14}$

Oestrogen receptor (ER) status in breast cancer has a major role in terms of predicting response to chemotherapy. Thus, according to the international breast cancer group, patients with ER negative breast cancer, receiving cyclophosphamide, methotrexate and 5 -florouracil have $48 \%$ reduction in their risk of recurrence and a $49 \%$ reduction in the risk of death from any cause. For patients with ER positive breast cancer both risks were reduced by only $1 \% .{ }^{15}$ In 2006, Berry et al also showed that, twice weekly doxorubicin/cyclophosphamide plus paclitaxel lowers the rate of recurrence and death by more than $50 \%$ in ER-negative breast cancer. ${ }^{16}$ In the neo-adjuvant setting, tumours without hormone receptors are more sensitive to chemotherapy than are hormone-sensitive tumours, with markedly higher rates of pathological complete response. ${ }^{17-19}$ Patients with progesterone $(\mathrm{PR})$ negative breast cancer do not have good response to chemotherapy compared to ER negative breast cancers according to MacGrogan et al. ${ }^{20}$ Meanwhile a study done by Colleoni et al. had shown that tumours of low expression of PR had a significantly higher response to primary chemotherapy. ${ }^{21}$

Triple negative breast cancer (ER-negative, PR-negative, HER2/neu not over expressed) is the absence of histological staining of ERs, PRs and HER2 in a breast cancer tissue. ${ }^{22}$ The triple negative breast cancer (TNBC) is reported to respond to neo-adjuvant chemotherapy ${ }^{23,24}$, but has a poor prognosis compared with non-TNBC (hormone receptor positive) patients. ${ }^{25}$ In evaluating response to neo-adjuvant chemotherapy the MD Anderson Centre in the U.S.A from 1985 to 2005 studied the response and prognosis of 1,118 TNBC and non-TNBC patients receiving neo-adjuvant chemotherapy. The pathological complete response rate in the $23 \%$ of non-TNBC patients was double that of TNBC patients. The overall 3-year freedom from progression was $63 \%$ in the TNBC and $76 \%$ in the non-TNBC groups, and the 3 year overall survival between the groups was $74 \%$ and
$89 \%$, respectively, confirming the relatively poor prognosis in TNBC. ${ }^{25}$

Published data on breast cancer in Ghana is scarce and for that matter knowledge on the breast cancer receptors and its association with chemotherapy response in Ghanaian women can hardly be found. This study sought to investigate the association between ER, PR and HER2/neu receptors status and response of breast cancer to chemotherapy given at the Komfo Anokye Teaching Hospital (KATH) in Kumasi, Ghana.

\section{Methods and Materials}

\section{Study design}

This study was a retrospective cohort study of patients with breast cancer who received chemotherapy at the oncology directorate of KATH.

\section{Study site}

The two cancer centres in Ghana are located in the two biggest cities: Accra which is the capital city and Kumasi which is the second largest city. Kumasi is located at the central part of Ghana. The KATH cancer centre serves about 6 out of the 10 regions in the country. The centre is the referral destination of almost all cancer cases seen at KATH. All breast cancer patients referred for neo-adjuvant chemotherapy, adjuvant chemotherapy or radiotherapy are seen at the cancer centre. After treatment they are subsequently followed up at the centre. The oncology centre in Kumasi has a clinic based registry which started in 2004. On the average 250 oncological cases are seen every year. Cervical cancer is the leading cancer recorded, followed by breast cancer.

\section{Study Procedure}

Patients who had a primary diagnosis of breast cancer as evidenced by a biopsy and receiving treatment at the oncology directorate of the KATH formed the study population. To be part of this study, patients should have been on the neo-adjuvant regimen, had taken four cycles (3 weekly) and with TNM stage T3-T4B, N0-N2, and M0. Patient's with co-morbidities: Hypertension, diabetes, asthma, had breaks in receiving chemotherapy or previous pathology on the breast other than breast cancer were excluded from the study.

Adriamycin $\left(60 \mathrm{mg} / \mathrm{m}^{2}\right)$ and cyclophosphamide $\left(600 \mathrm{mg} / \mathrm{m}^{2}\right)$ through intravenous bolus injections is the standard neo-adjuvant chemotherapy given at the centre. Patients with incomplete clinical response or no response at all after first line Adriamycin and cyclophosphamide were offered a second line paclitaxel $170 \mathrm{mg} / \mathrm{m}^{2}$ I.V route plus capecitabine 
$1000 \mathrm{mg} / \mathrm{m}^{2}$ oral. The Response Evaluation Criteria In Solid Tumour (RECIST 1.0) was used to measure clinical response. ${ }^{26}$ Clinical examination using ruler/tape measure was used to assess the tumor size before and after chemotherapy. Between 2009 and 2012, 400 breast cancer cases were seen, 210 of them had received neo-adjuvant chemotherapy. Out of these 91 patients qualified for inclusion into the study. Reference numbers on the patients chart were used to trace the pathology reports per patient from the pathology department. Report included histological description of the tumour, macro and micro descriptions and ER/PR, HER2/neu results.

\section{Data capture}

A pre-designed data collection sheet capturing information such as the menopausal status, parity, age, histological type ER, PR, HER2/neu expression and treatment history was used to capture data from the patients' charts. An electronic database designed using Epiinfo version 3.2.2 to capture all the information on the datasheet was designed and the data double entered. This was compared on regular basis to identify anomalous data and figures and corrections made.

\section{Statistical analysis}

Statistical analysis was carried out using Stata SE 12.1. The prevalence of the various receptors and treatment outcomes were analysed and presented as proportions with their binomial exact confidence intervals. Trends in the various associated parameters such as the age grouping and remission status were determined using the chi-square test for trend. To determine the relationship between the various receptor status and outcome, an age adjusted odds ratios (OR) with their $95 \%$ confidence intervals and associated p-values were determined using a logistic regression as receptor status and outcome of the first line were both associated with age. For all analysis a two-sided p-value of $<0.05$ was considered statistically significant.

\section{Ethical considerations}

Ethical Permission was granted by the Committee of Human Research Publication and Ethics, Kwame Nkrumah University of Science and Technology, Kumasi, Ghana.

\section{Results}

In all 91 patients with breast cancer were included in the study. All patients completed the first line treatment successively with 36 (38.3\%, 95\% CI: 28.5 to 48.9$)$ going into complete remission (Figure 1). The rest were then put through second line treatment with 34 (58.6\%, 95\% CI: 44.9 to 71.4$)$ of them going into complete remission. After both first and second line treatment a total of 70 (74.5\%, 95\% CI: 64.4 to 82.9) had gone into complete remission.

Most 58 (61.7\%) were between 41 and 60 years with the youngest being 30 years and the oldest 76 years (Table 1 ). The age grouping showed an increasing trend $(\mathrm{p}=0.002)$ towards better chance of remission after first line treatment with proportions of those going into remission being $5.6 \%$ (1/18), $43.1 \%(25 / 58)$ and $55.6 \%$ (10/18) for the $30-40$ years, 41-60 years and 61-76 years groups respectively. Complete remission after second line treatment was not associated with age (trend $\mathrm{p}=0.259$ ) though it decreased with increasing age with proportions going into of complete remission of $70.6 \%$ (12/17), $54.6 \%(18 / 33)$ and $50.0 \%(4 / 8)$ for the age ranges of 30-40 years, $41-60$ years and $61-76$ years groups respectively.

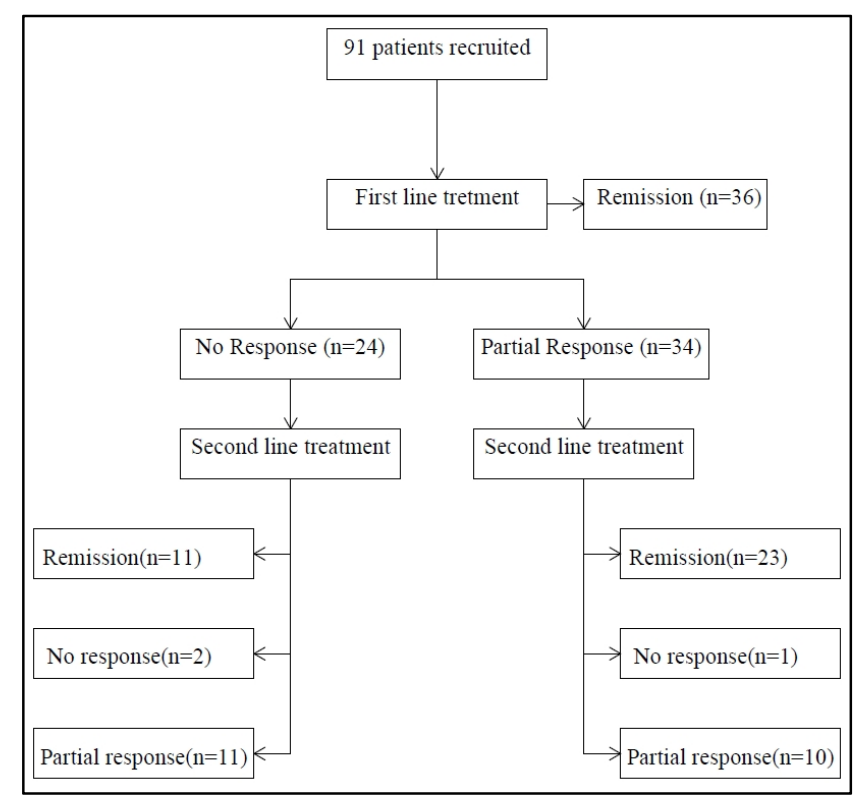

FIG. 1: Flow chart of patient treatments and outcomes.

The mean (SD) parity of the patients was 4.1 (1.8) with most (44, 46.8\%) having 3 to 4 children. Also most of the patients were pre-menopausal.

The prevalence of ER, PR, HER2/neu and TNBC were 34.0\% (95\% CI: 24.6 to 44.5 ), $20.2 \%$ (95\% CI: 12.6 to 29.7$), 8.5 \%$ (95\% CI: 3.7 to 16.1 ) and 59.6\% (95\%CI: 48.9 to 69.6$)$ respectively. All patients with PR positive breast cancer also were ER positive. No significant relationship in terms of trend or difference in proportions between parity and menopausal status on one hand and the presence or absence of the receptors was detected (Table 1). However there was a significant trend $(\mathrm{p}=0.036)$ of the proportion of patients with ER getting higher with increasing age. Also TNBC was marginally $(p=0.095)$ related to age with it being more common in younger age groups. 
TABLE 1: Distribution of age, parity, menopausal stage and receptor status.

\begin{tabular}{ccccccc}
\hline \hline & & \multicolumn{5}{c}{ Hormone/Receptor status n (\%) } \\
\cline { 5 - 6 } Characteristic & $\mathrm{N}(\%)$ & ER & PR & HER2/neu & TNBC \\
\hline Age (yrs) & & & & & \\
$30-40$ & $18(19.1)$ & $3(17.7)$ & $2(11.1)$ & $1(5.6)$ & $14(77.8)$ \\
$41-60$ & $58(61.7)$ & $20(34.5)$ & $12(20.7)$ & $7(12.1)$ & $33(56.9)$ \\
$61-76$ & $18(19.2)$ & $9(50.0)$ & $5(27.8)$ & $0(0.0)$ & 9 & $(50.0)$ \\
\hline$p$-value (proportion) & & 0.107 & 0.456 & 0.244 & 0.189 \\
$p$-value (trend) & & 0.036 & 0.216 & 0.552 & 0.091 \\
\hline Parity & & & & & \\
$0-2$ & $14(14.9)$ & $6(42.9)$ & $4(28.6)$ & $2(14.3)$ & $7(50.0)$ \\
$3-4$ & $44(46.8)$ & $16(36.4)$ & $10(22.7)$ & $3(6.8)$ & $26(59.1)$ \\
$>=5$ & $36(38.3)$ & $10(27.8)$ & $5(13.9)$ & $3(8.3)$ & $23(63.9)$ \\
\hline$p$-value (proportion) & & 0.544 & 0.433 & 0.683 & 0.665 \\
$p$-value (trend) & & 0.274 & 0.202 & 0.642 & 0.381 \\
\hline Menopausal status & & & & & \\
Pre-menopause & $46(48.9)$ & $12(26.1)$ & $7(15.2)$ & $5(10.9)$ & $30(65.2)$ \\
Menopause & $14(14.9)$ & $5(35.7)$ & $2(14.3)$ & $0(0.0)$ & $9(64.3)$ \\
Post-menopause & $34(36.2)$ & $15(44.1)$ & $10(29.4)$ & $3(8.8)$ & $17(50.0)$ \\
\hline$p$-value (proportion) & & 0.240 & 0.247 & 0.441 & 0.362 \\
$p$-value (trend) & & 0.244 & 0.129 & 0.694 & 0.181 \\
\hline \hline
\end{tabular}

TABLE 2: Relationship between tumor staging and receptor status.

\begin{tabular}{ccccc}
\hline \hline & & \multicolumn{3}{c}{ Receptor n (\%) } \\
\cline { 4 - 5 } Marker/receptor & ER & PR & HER2/neu & TNBC \\
\hline Nodal staging & & & & \\
N0 & $5(15.6)$ & $2(10.5)$ & $1(12.5)$ & $7(12.5)$ \\
N1 & $19(59.4)$ & $13(68.4)$ & $2(25.0)$ & $24(42.9)$ \\
N2 & $8(25.0)$ & $29(21.1)$ & $4(50.0)$ & $18(32.1)$ \\
N3 & $0(0.0)$ & $0(0.0)$ & $0(0.0)$ & $7(12.6)$ \\
T- staging & & & & \\
1 & $0(0.0)$ & $0(0.0)$ & $0(0.0)$ & $1(1.8)$ \\
2 & $5(15.6)$ & $3(15.8)$ & $0(0.0)$ & $10(17.9)$ \\
3 & $5(15.6)$ & $0(0.0)$ & $2(25.0)$ & $5(8.9)$ \\
$4 \mathrm{~b}$ & $15(46.9)$ & $11(57.9)$ & $4(50.0)$ & $25(44.6)$ \\
$4 \mathrm{c}$ & $5(15.6)$ & $4(21.1)$ & $2(25.0)$ & $14(25.0)$ \\
$4 \mathrm{~d}$ & $2(6.3)$ & $1(5.3)$ & $0(0.0)$ & $1(1.8)$ \\
Grade & & & & \\
1 & $5(15.6)$ & $1(5.3)$ & $2(25.0)$ & $4(7.1)$ \\
2 & $21(65.6)$ & $14(73.7)$ & $3(37.5)$ & $18(32.2)$ \\
3 & $6(18.8)$ & $4(21.0)$ & $3(37.5)$ & $34(60.7)$ \\
\hline \hline
\end{tabular}

TABLE 3: Relationship between receptor status and post treatment complete remission.

\begin{tabular}{|c|c|c|c|c|}
\hline \multirow[b]{2}{*}{ Marker/receptor status } & \multicolumn{2}{|c|}{ N (\%) } & \multirow[b]{2}{*}{$\mathrm{OR}^{*}(95 \% \mathrm{CI})$} & \multirow[b]{2}{*}{ p-value } \\
\hline & Non-remission & Remission & & \\
\hline \multicolumn{5}{|c|}{ First line treatment } \\
\hline Estrogen & $7(12.1)$ & $25(69.4)$ & $15.7(5.2-47.3)$ & $<0.001$ \\
\hline Progesterone & $4(6.9)$ & $15(41.7)$ & $9.4(2.7-33.4)$ & 0.001 \\
\hline Her2Neu & $5(8.6)$ & $3(8.3)$ & $1.1(0.2-5.3)$ & 0.868 \\
\hline TNBC & $47(81.0)$ & $9(25.0)$ & $0.1(0.0-0.2)$ & $<0.001$ \\
\hline \multicolumn{5}{|c|}{ Second line treatment } \\
\hline Estrogen & $6(25.0)$ & $1(2.9)$ & $0.1(0.0-0.8)$ & 0.033 \\
\hline Progesterone & $4(16.7)$ & $0(0.0)$ & - & - \\
\hline Her2Neu & $3(12.5)$ & $2(5.9)$ & $0.4(0.1-2.7)$ & 0.363 \\
\hline TNBC & $16(66.7)$ & $31(91.2)$ & $5.2(1.2-22.5)$ & 0.027 \\
\hline \multicolumn{5}{|c|}{ Combined first \& second line treatment } \\
\hline Estrogen & $6(25.0)$ & $26(37.1)$ & $1.6(0.6-4.7)$ & 0.380 \\
\hline Progesterone & $4(16.7)$ & $15(21.4)$ & $1.3(0.4-4.3)$ & 0.722 \\
\hline Her2Neu & $3(12.5)$ & $5(7.1)$ & $0.6(0.1-2.5)$ & 0.451 \\
\hline TNBC & $16(66.7)$ & $40(57.1)$ & $0.7(0.3-2.0)$ & 0.531 \\
\hline
\end{tabular}

${ }^{*} \mathrm{OR}$ adjusted for age 
The presence of ER or PR were good predictors of remission after the first line treatment with respective age adjusted odds of 15.7 (95\% CI: 5.2 to 47.3, p < 0.001) and 9.4 (95\% CI: 2.7 to $33.4, \mathrm{p}=0.001)$. TNBC on the other hand predicted significantly poor outcome (OR: $0.1,95 \% \mathrm{CI}$ : 0.0 to 0.2 , p < 0.001) after failed first line treatment (Table 3 ). This relationship seem to be reversed in those who went ahead to receive the second line treatment. Here, none of the $4 \mathrm{PR}$ receptor positive persons went into remission. Also ER positivity was a predictor of poorer prognosis with an age adjusted OR of 0.1 (95\% CI: 0.0 to $0.8, p=0.033)$. On the other hand TNBC was associated with a better prognosis with an age adjusted OR of 5.2 (95CI: 1.2 to $22.5, \mathrm{p}=0.027$ ). There was no significant relationship observed between HER2/neu and the response to chemotherapy. Also after combined first and second line treatment outcomes, presence or absence of any receptor status was not a predictor of remission.

\section{Discussion}

The prevalence of ER from our study was $34.0 \%$. In a study done by Ohene Yeboah and Adjei, of 68 breast cancer cases 47\% were ER positive. ${ }^{27}$ Yanney et al. 2008 also showed 43\% ER positivity in 74 tumours. ${ }^{28}$ These two studies in addition to our findings seem to show that ER positive breast cancer may be on a low side in Ghana. The use of tamoxifen (hormonal therapy) targets ER; and it has been shown to reduce breast cancer mortality by about a third throughout the last 15 years. ${ }^{29}$ Low prevalence of ER positive breast cancer may have a negative impact on the prognosis of the Ghanaian women with breast cancer.

Eighty four percent of the patients with breast cancer who were ER positive had lymph node involvement (Table 2) with all being either grade 2 or 3 . These findings may support the fact that breast cancers among people of African descent are more aggressive histologically and clinically. ${ }^{30}$

Proportion of pre-menopausal patients with progesterone positivity was lesser than post-menopausal patients in our study. Perhaps a larger sample size as the study done by Clark et al. would be needed to make a fair comparison. Similar to ER, PR positivity predicts a good response to first line Adriamycin and cyclophosphamide according to our study. Allegra in his study showed that ER and PR were important predictors of response to chemotherapy in breast cancer. ${ }^{31}$

HER2/neu immuno-expression was positive in $67.8 \%$ of breast cancer in people of the middle Euphrates region of Iraq. ${ }^{32} 17.5 \%$ of the HER2/neu positive tumours was also found in Jordan in a study done by Sughayer et al. in 2006. ${ }^{33} \mathrm{With} 46$ Japanese patients, Sawaki et al. recorded a proportion of $50 \%$ HER2/neu positive tumours. ${ }^{34}$ Ohene Yeboah and Agyei found 20.3\% of HER2/neu positive breast cancer patients in Ghana. We report of $8.5 \%$ of HER2/neu in our study. Seven percent of patients in our study who were HER2/neu positive had lymph node positive disease. According to Tokatli et al. in 2005 there is a significant association between HER2/neu and increasing number of involved nodes. $^{35}$ They evaluated the prognostic significance of HER2/neu over expression in hormone receptor and axillary lymph node positive breast cancer in a single institution and concluded that for node positive patients HER2/neu over expression was a significant predictor of disease free survival.

Coupled with our study it may possibly imply that the HER2/neu expression in node positive breast cancer patients in Ghana is low. Thus the majority of breast cancer patients seen in Ghana have nodal positive disease, but the low HER2/neu expressions may relatively predict a good prognosis.

HER2/neu expression and inexpression in breast cancer tumours does not seem to have any effect on chemotherapy response, probably because of its low prevalence in our study. To define the effect of HER2/neu oncogene overexpression on sensitivity to chemotherapeutic drugs, Pegram et al ${ }^{36}$ did not find any difference in terms of response to chemotherapy between tumours with and without HER2/neu expression. We found similar results in our study; HER2/neu did not favour good or no response to first line Adriamycin and cyclophosphamide. This implies that HER2/neu may not be used as a predictive marker for first line chemotherapy response.

\section{Conclusion}

Most patients with breast cancer in Kumasi are TNBC with about a third being ER positive. HER2/Neu overexpression was relatively uncommon. ER and PR positivity portends good prognosis after first line treatment but for patients who do not go into remission also do not do well on second line treatment. On the other hand persons with TNBC had poorer outcome after first line treatment but better outcome in terms of remission after the second line treatment. HER2/Neu overexpression was not associated with complete remission in our population.

\section{Conflict of interest}

The authors declare that they have no conflicts of interest. The authors alone are responsible for the content and writing of the paper.

\section{Acknowledgement}

The authors would like to thank the staff of oncology department (KATH) for their assistance and support. 


\section{References}

1. Jemal A, Bray F, Center MM, et al. Global cancer statistics. CA Cancer J Clin 2011; 61:69-90.

2. Parkin DM, Bray F, Ferlay J, Pisani P. Global cancer statistics, 2002. CA Cancer J Clin 2005; 55:74-108.

3. Mettlin C. Global breast cancer mortality statistics. CA Cancer J Clin 1999; 49:138-44.

4. Parkin DM. Cancer in developing countries. Cancer Surv 1994; 19-20:519-61.

5. Clegg-Lamptey J, Hodasi W. A study of breast cancer in korle bu teaching hospital: assessing the impact of health education. Ghana Med J 2007; 41:72-7.

6. Clegg-Lamptey JN, Dakubo JC, Attobra YN. Psychosocial aspects of breast cancer treatment in Accra, Ghana. East Afr Med J2009; 86:348-53.

7. Ohene-Yeboah MO. An audit of excised breast lumps in Ghanaian women. West Afr J Med 2005; 24:252-5.

8. Barret-Lee PJ. Growth factor signalling in clinical breast cancer and its impact on response to conventional therapies: a review of chemotherapy. Endocr Relat Cancer 2005; 12 Suppl 1: S125-33.

9. Muss HB, Thor AD, Berry DA, et al. c-erbB-2 expression and response to adjuvant therapy in women with node-positive early breast cancer. $N$ Engl J Med 1994, 330:1260-6.

10. Slamon DJ, Clark GM, Wong SG, et al. Human breast cancer: correlation of relapse and survival with amplification of the HER-2/neu oncogene. Science 1987; 235:177-82.

11. Willsher PC, Pinder SE, Gee JM, et al. C-erbB2 expression predicts response to preoperative chemotherapy for locally advanced breast cancer. Anticancer Res 1998; 18:3695-8.

12. Gregory RK, Powles TJ, Salter J, et al. Prognostic relevance of cerbB2 expression following neoadjuvant chemotherapy in patients in a randomised trial of neoadjuvant versus adjuvant chemoendocrine therapy. Breast Cancer Res Treat 2000; 59:171-5.

13. Thor AD, Berry DA, Budman DR, et al. erbB-2, p53, and efficacy of adjuvant therapy in lymph node-positive breast cancer. I Natl Cancer Inst 1998; 90:1346-60.

14. Ellis IO, Dowsett M, Bartlett J, et al. Recommendations for HER2 testing in the UK. J Clin Pathol 2000; 53:890-2.

15. International Breast Cancer Study Group (IBCSG). Endocrine responsiveness and tailoring adjuvant therapy for postmenopausal lymph node-negative breast cancer: a randomized trial. J Natl Cancer Inst 2002; 94:1054-65.
16. Berry DA, Cirrincione C, Henderson IC, et al. Estrogen-receptor status and outcomes of modern chemotherapy for patients with node-positive breast cancer. Jama 2006; 295:1658-67.

17. Colleoni M, Viale G, Zahrieh D, et al. Chemotherapy is more effective in patients with breast cancer not expressing steroid hormone receptors: a study of preoperative treatment. Clin Cancer Res 2004; 10:6622-8.

18. Gianni L, Zambetti M, Clark K, et al. Gene expression profiles in paraffin-embedded core biopsy tissue predict response to chemotherapy in women with locally advanced breast cancer. J Clin Oncol 2005; 23: 7265-77.

19. Buzdar AU, Valero V, Theriault RL, et al. Pathological complete response to chemotherapy is related to hormone receptor status [abstract]. Breast Cancer Res Treat 2003; 85:2.

20. MacGrogan G, Mauriac L, Durand M, et al. Primary chemotherapy in breast invasive carcinoma: predictive value of the immunohistochemical detection of hormonal receptors, p53, c-erbB-2, MiB1, pS2 and GST pi. Br J Cancer 1996; 74: 1458-65.

21. Colleoni M, Orvieto E, Nolé F, et al. Prediction of response to primary chemotherapy for operable breast cancer. Eur J Cancer 1999; 35: 574-9.

22. Irvin WJ Jr, Carey LA. What is triple-negative breast cancer? Eur J Cancer 2008; 44:799-805.

23. Rouzier R, Perou CM, Symmans WF, et al. Breast cancer molecular subtypes respond differently to preoperative chemotherapy. Clin Cancer Res 2005; 11:5678-85.

24. Carey LA, Dees EC, Sawyer L, et al. The triple negative paradox: primary tumor chemosensitivity of breast cancer subtypes. Clin Cancer Res 2007; 13: 2329-34.

25. Liedtke C, Mazouni C, Hess KR, et al. Response to neoadjuvant therapy and long-term survival in patients with triple-negative breast cancer. J Clin Oncol 2008; 26:1275-81.

26. Therasse P, Arbuck SG, Eisenhauer EA, et al. New guidelines to evaluate the response to treatment in solid tumors. European Organization for Research and Treatment of Cancer, National Cancer Institute of the United States, National Cancer Institute of Canada. J Natl Cancer Inst 2000; 92: 205-16.

27. Ohene-Yeboah M, Adjei E. Breast cancer in $\mathrm{Ku}-$ masi, Ghana. Ghana Med J2012; 46: 8-13.

28. Christina Davies, Hongchao Pan, Jon Godwin, et al. and for the Adjuvant Tamoxifen: Longer Against Shorter (ATLAS) Collaborative Group $†$ Long-term effects of continuing adjuvant tamoxifen to 10 years versus stopping at 5 years after diagnosis of oestrogen receptor-positive breast cancer: ATLAS, a randomised trial 2013; 381:805-16. 
29. Yarney J, Vanderpuye V, Clegg Lamptey JN. Hormone receptor and Her-2 expression in breast cancers among sub-Saharan African women. Breast $J$ 2008; 14: 510-11.

30. Fregene A, Newman LA. Breast cancer in sub-Saharan Africa: how does it relate to breast cancer in African-American women? Cancer 2005; 103:1540-50.

31. Allegra JC, Lippman ME, Simon R, et al. Association between steroid hormone receptor status and disease-free interval in breast cancer. Cancer Treat Rep 1979; 63:1271-7.

32. Al-Dujaily EA, Al-Janabi AA, Pierscionek T, Yasseen AA. High prevalence of HER-2/neu overexpression in female breast cancer among an Iraqi population exposed to depleted uranium. J Carcinog 2008; 7:8.

33. Sughayer MA, Al-Khawaja MM, Massarweh S, Al-Masri M. Prevalence of hormone receptors and HER2/neu in breast cancer cases in Jordan. Pathol Oncol Res 2006; 12:83-6.
34. Sawaki M, Ito Y, Akiyama F, et al. High prevalence of HER-2/neu and p53 overexpression in inflammatory breast cancer. Breast Cancer 2006; 13:172-8.

35. Tokatli F, Altaner S, Uzal C, et al. Association of HER-2/neu overexpression with the number of involved axillary lymph nodes in hormone receptor positive breast cancer patients. Exp Oncol 2005; 27:145-9.

36. Pegram MD, Finn RS, Arzoo K, et al. The effect of HER-2/neu overexpression on chemotherapeutic drug sensitivity in human breast and ovarian cancer cells. Oncogene1997; 15:537-47. 